\title{
Solitary Skull Metastasis as Initial Manifestation of Hepatocellular Carcinoma - A Case Report
}

\author{
Ellyda $M N^{a}$ and Mohd Shafie $A^{b}$
}

\begin{abstract}
a Department of Radiology, International Islamic University Malaysia, Kuantan, Pahang, Malaysia.

b Department of Radiology, School of Medical Sciences, Universiti Sains Malaysia, Kubang Kerian, Kelantan, Malaysia.
\end{abstract}

\begin{abstract}
Metastatic spread of tumors to the skull is quite unusual and often represents diagnostic and therapeutic issues. Skull involvement can be observed in various neoplasms of epithelial origin and are most often due to lung, breast, thyroid, kidney and prostate cancers. However, skull metastases from hepatocellular carcinoma (HCC) have been rarely reported. The prognosis for patients with hepatocellular carcinoma is so poor that treatment of such distant metastatic lesion cannot be achieved before death occurs due to the primary malignancy. Therefore, the clinical manifestations of cranial metastasis prior to that of primary hepatocellular carcinoma have rarely been reported. This case illustrates a rare case of skull metastasis as an initial manifestation of hepatocellular carcinoma. Although a solitary skull metastasis prior to the diagnosis of HCC demonstrates rare metastatic behavior for HCC, especially in Asia, skull metastases from HCC should be included in the differential diagnosis of skull tumors, even if the patient is asymptomatic of liver cirrhosis.
\end{abstract}

KEYWORDS: Hepatocellular carcinoma, Skull metastasis, Chemoembolization

\section{CASE REPORT}

A 61 year-old Malay male with underlying history of diabetes, hypertension and Hepatitis B presented with painless subcutaneous swelling in the scalp of 4 months duration. Physical examination revealed signs of chronic liver disease with multiple left supraclavicular lymphadenopathy. No hepatosplenomegaly. A well defined $14.0 \mathrm{~cm} \times 12.0 \mathrm{~cm}$ swelling in the right parietal scalp which was firm in consistency and non tender was present. No pus discharge was noted from the swelling. His serum alpha fetoprotein was high (483.9 $\mathrm{IU} / \mathrm{mL}$ ). Skull radiograph followed by non contrast and contrasted computed tomography (CT) scan of the brain were done. Skull radiograph showed an osteolytic lesion in the right parietal bone (Figure 1). CT brain findings revealed soft tissue mass at the right parietal region with adjacent bony destruction (Figure 2). Subsequently, a percutaneous needle biopsy of the scalp lesion confirmed a metastasis from a multicentric hepatocellular carcinoma. A retrograde diagnostic work-up for detecting the primary cancer was then performed. An abdominal CT scan revealed

Corresponding author;

Dr Ellyda Muhammed Nordin,

Radiology Department,

International Islamic University Malaysia,

Jalan Hospital, 25100 Kuantan,

Pahang Darul Makmur, Malaysia.

Email: elly7575@gmail.com multiple ill-defined hypodense liver lesions (Figure 3) consistent with hepatocellular carcinoma with bilateral adrenal metastases (Figure 4).

\section{DISCUSSION}

Hepatocellular carcinoma (HCC) is one of the most common cancers worldwide and the most common primary malignant tumor of the liver. The major risk factor for HCC is Hepatitis C, evidenced by presence of antibody to HCV (anti-HCV) and HCV RNA. ${ }^{1}$ Other risk factors include liver cirrhosis due to long standing chronic hepatitis B, C, or hereditary hemochromatosis and other metabolic disorders. Patients with longstanding alcoholic cirrhosis are also at risk for developing this tumor. ${ }^{1}$

HCC spreads locally and metastasizes distally by hematogenous and lymphatic routes. ${ }^{1}$ The commonest site for spread of HCC is via invasion of the liver capsule followed by extra vascular invasion, vascular invasion and lastly by intrahepatic metastasis. ${ }^{2}$ It is been reported that extra-hepatic spreading of HCC is not uncommon and usually involve lymph nodes (42\%), lungs (18\%) and the adrenal gland. ${ }^{1,3}$

This patient initially visited the hospital due to the incidentally discovered scalp mass. It is a similar presentation to the reported case by Latif $\mathrm{A}$ et al in 2002. From the previous literature, metastases from $\mathrm{HCC}$ is commonly present in the skull base and less 
frequently seen in the skull vault with a subcutaneous mass as the most common clinical presentation (63\%). Other signs and symptoms are neurological deficits (44\%), headache $(11 \%)$ and seizures. ${ }^{1,4}$ Patients who present with neurological deficits usually manifest as facial palsy, deafness, visual disturbance, facial numbness, weakness of limbs, and other cranial nerve palsies, depending on the size of tumor and its location. ${ }^{1,4}$

HCC rarely metastasizes to the bones. ${ }^{5,6}$ Such osseous metastases are mainly of the spine or long skeletal bones, and only $6 \%$ to the skull bones in autopsy cases. ${ }^{7}$ The incidence of intracranial metastases and/ or skull metastases is extremely rare and assessed on the basis of postmortem studies. In all cases the plain skull radiograph appearance of the metastases was osteolytic. ${ }^{8}$

Recently, diagnosis is reached by neurological findings and imaging studies, such as CT and MRI because of improvements in these modalities. The metastatic lesions appeared as expansile osteolytic masses on CT and as hypervascular masses on angiography. On MR imaging, compared with the brain parenchyma, the lesions were iso- or hypointense on T1-weighted and T2-weighted MR images. The lesions were moderately to markedly enhanced by intravenous gadoliniumDTPA. The relatively hypointense tumor on T2-weighted MR images associated with flow void, different from primary skull tumors or directly invasive tumors may support the diagnosis of HCC metastasis. ${ }^{9}$

Hepatocellular carcinoma is an aggressive tumor and may be at an advanced stage when detected. Treatment of hepatocellular carcinoma maybe directed towards a cure, or focused towards palliation. Recently, with the advances in non-surgical treatment of HCC worldwide, a number of therapies that offer good palliation can be choosen. 9 Treatment options are dictated by the extent and stage of the tumor and functional reserve of the liver. Among them are percutaneous ablation including percutaneous ethanol injection therapy (PEIT), radiofrequency ablation (RFA) and transcatheter embolization. All of these methods can prolong the survival rate of the patient even in advanced cases of HCC. However, they have their particu1ar indications, contraindications and complications. ${ }^{9}$

The prognosis for patients with HCC is so poor that treatment of such a distant metastatic lesion cannot be achieved before death occurs due to the primary malignancy. Therefore, the clinical manifestations of cranial metastasis prior to that of primary HCC have rarely been reported. ${ }^{9}$

As illustrated by this case, the diagnosis of HCC should be considered in the differential diagnosis of patients with subcutaneous scalp mass and osteolytic defects on radiograph especially in the patients with underlying cirrhosis. An isolated skull metastasis may be the sole initial presentation of HCC. Early diagnosis is essential in order to treat the primary disease. In conclusion, bone metastases from HCC are rare and it is even rarer to find bone metastases as the first overt manifestation of HCC. 


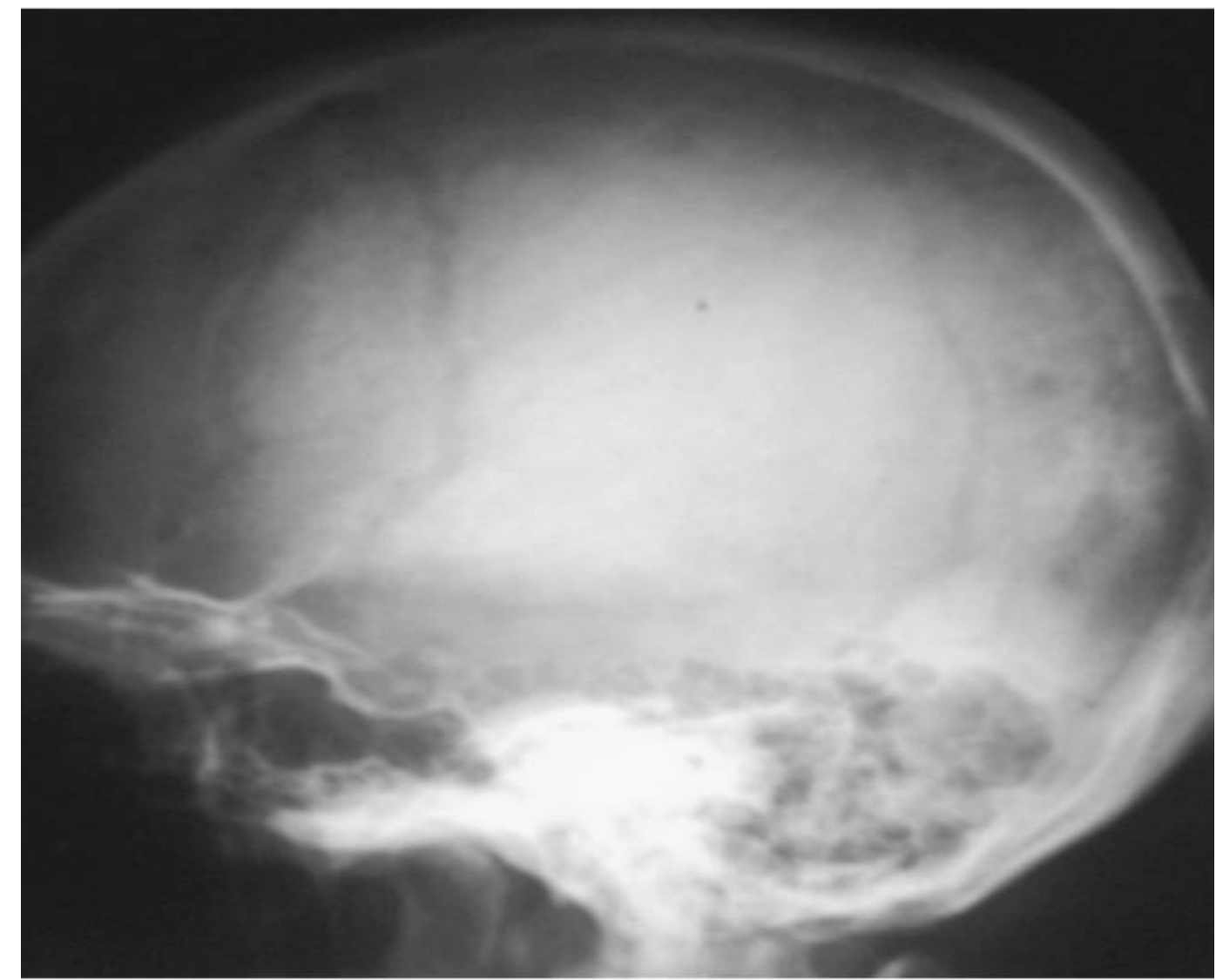

Figure 1. Lateral skull X-ray showed an osteolytic lesion in the right posterior parietal bone (arrows).

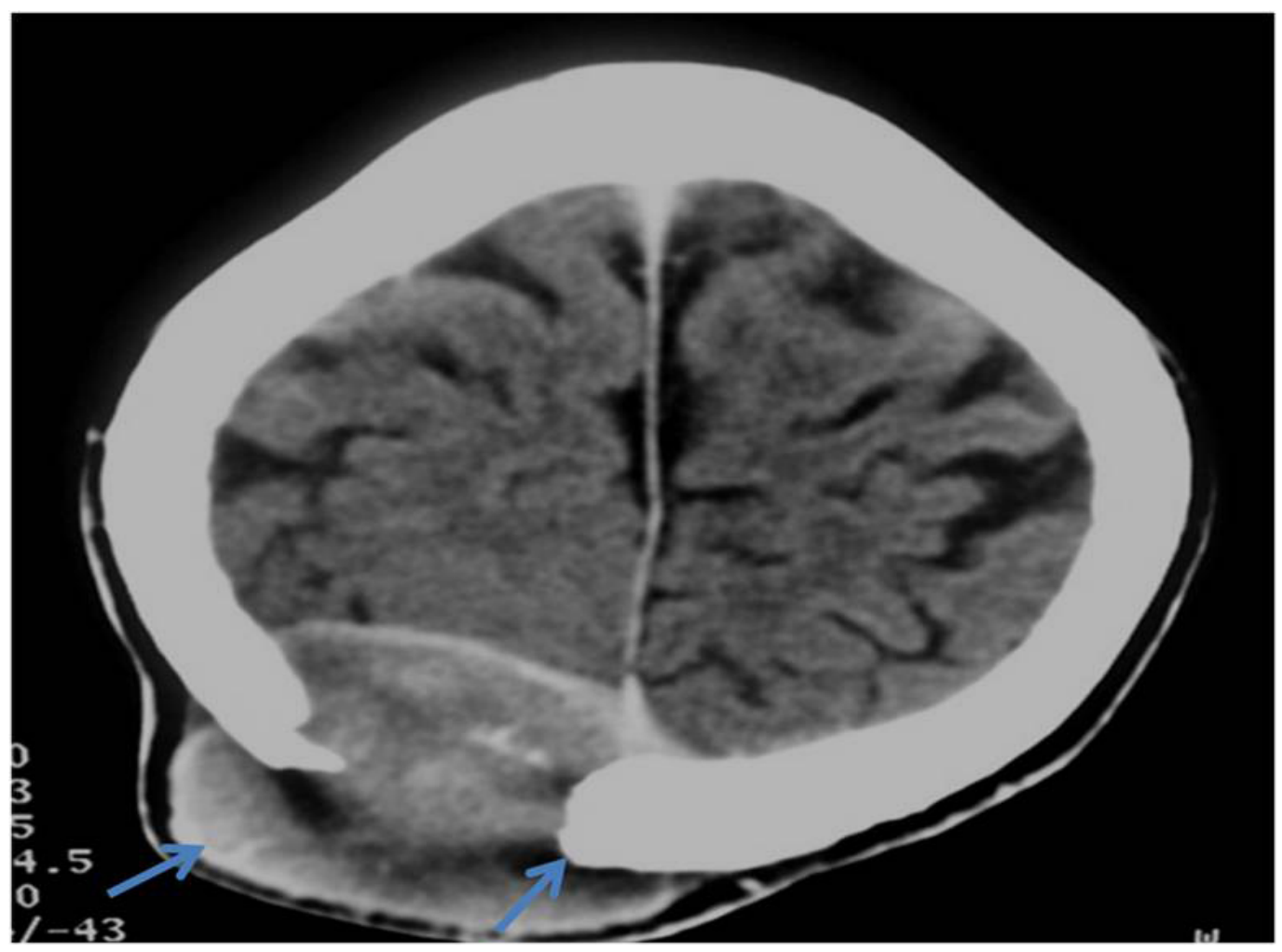

Figure 2. Contrast enhanced CT of the brain showed a well defined enhancing soft tissue mass in the right posterior parietal region with bony destruction (arrows). 


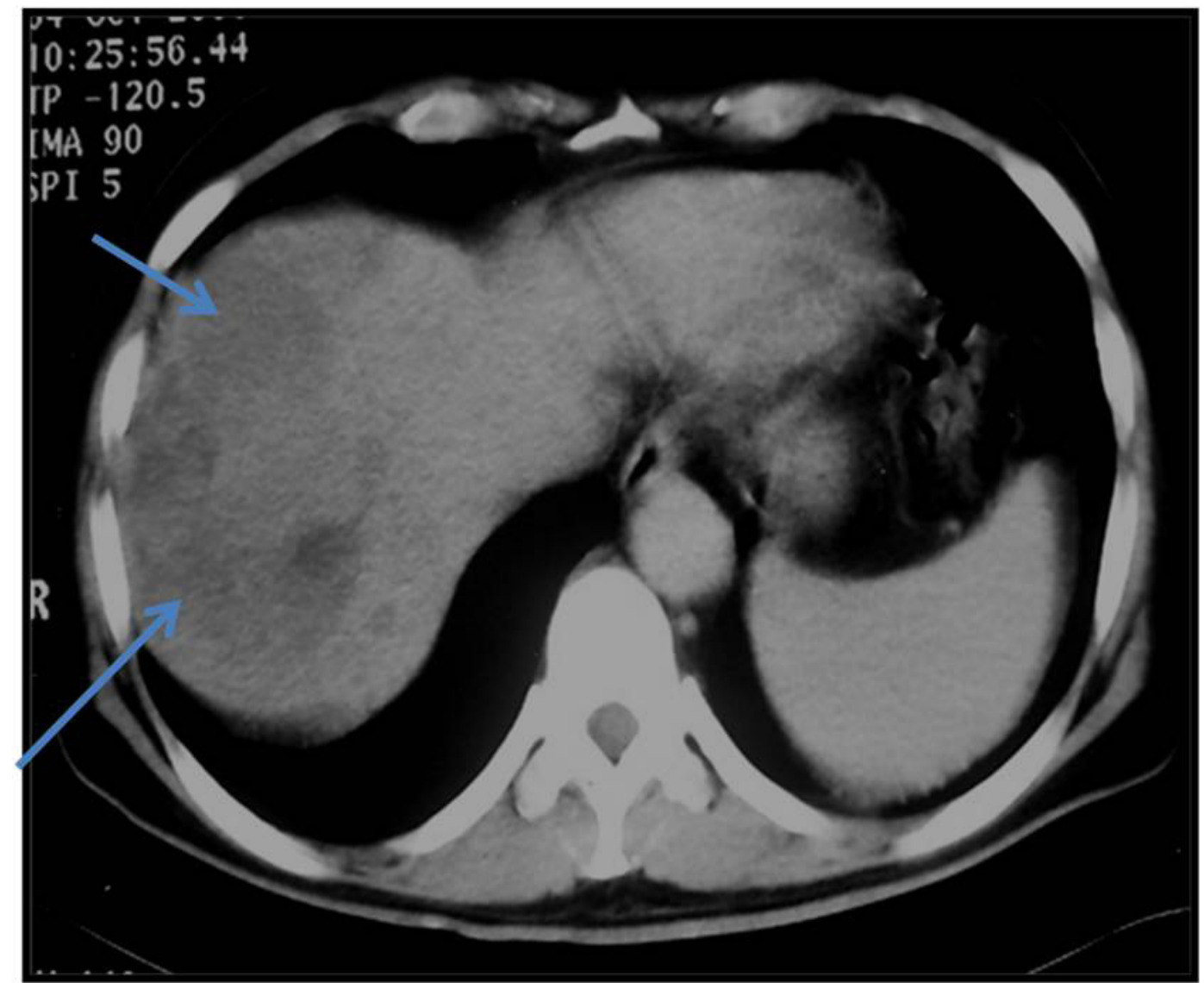

Figure 3. Contrast enhanced CT of abdomen showed multiple ill-defined area of heterogeneously hypodense lesion in both liver lobes with minimal enhancement on arterial phase (arrows).

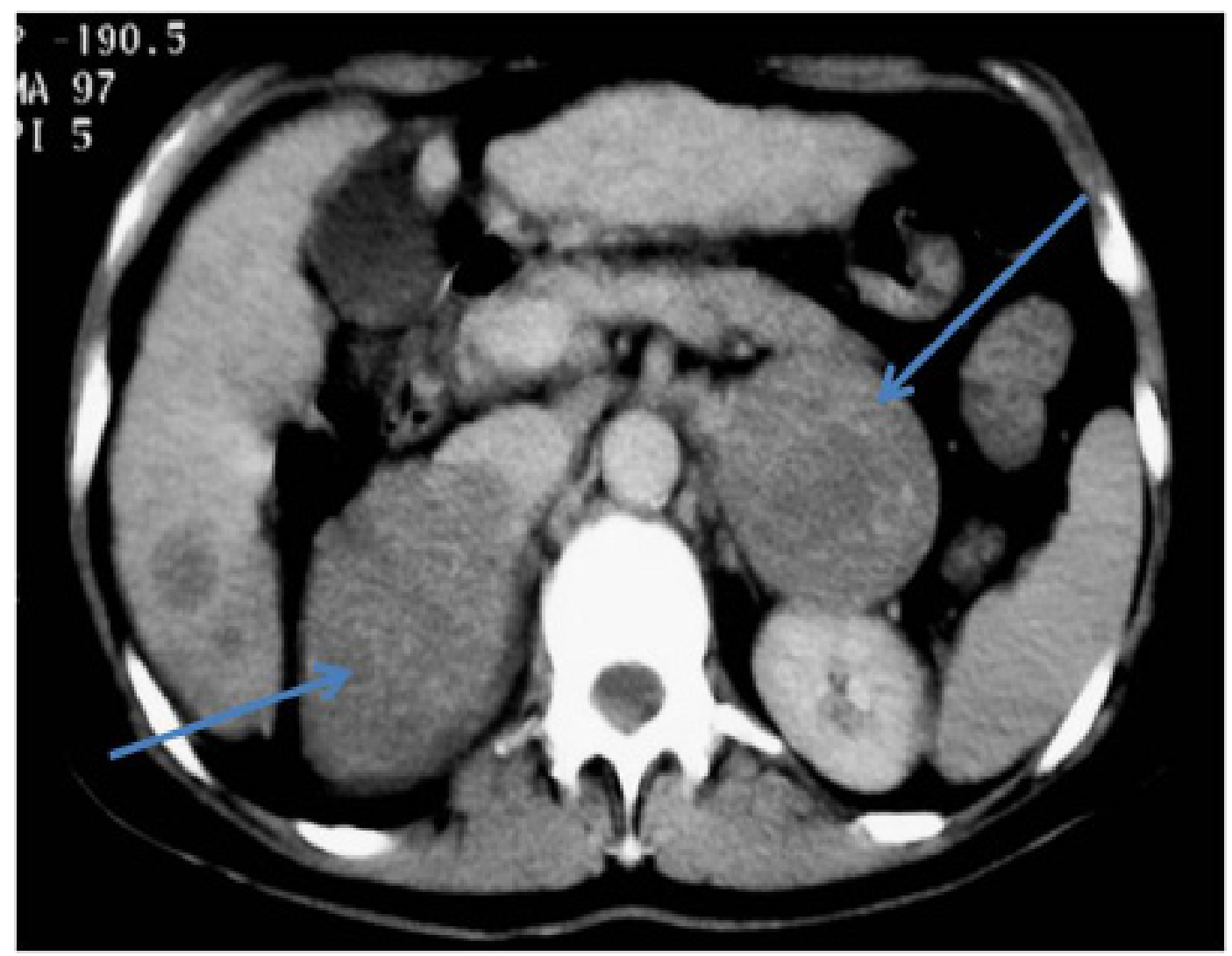

Figure 4. CECT showed enlarged and lobulated right and left adrenal glands with minimal enhancement in keeping with adrenal metastases (arrows). 


\section{REFERENCES}

1. Latif A, Muzrekchi A. Bone Metastasis Hepatocellular Carcinoma. QMJ 2002; 11(1)

2. Yen FS, Wo JC, Lai CR, et al. Clinical and Radiological pictures of Hepatocellular Carcinoma with intracranial metastasis. J Gastroenterol Hepatol 1995; 10:413

3. Szmuness W. Hepatocellular Carcinoma and the Hepatitis B virus: Evidence of a causal association. Prog Med Virol 1978; 24:40-69

4. Shim YS et al. Solitary skull metastasis as initial manifestation of hepatocellular Carcinoma. World J Surg Oncol 2008; 6:66

5. Kim SR, Kanda F, Kobessho H, et al. Hepatocellular carcinoma metastasizing to the skull base involving multiple cranial nerves. World J Gastroenterol 2006; 12:6727-29

6. Hiraide F. Metastatic tumor of the temporal bone presenting as sudden deafness. Jibi To Rinsho 1989; 35:1022-7 [Japanese]

7. Abramas H, Spiro R, Goldstein N. Metastases in carcinoma. Analysis of 1,000 autopsied cases. Cancer 1950; 3:74-81

8. Yanase $\mathrm{Y}$, Sano K, Hama H. Case report of a primary hepatoma with metastasis to the femur and statistical review. Kansai Denryoku Byoin Igaku Zasshi 1982; 4:94-9 [Japanese]

9. Kim SR et al. Hepatocellular carcinoma metastasizing to the skull base involving multiple cranial nerves. World J Gastroenterol 2006; 12:6727-29 
